ANNALES

POLONICI MATHEMATICI

$97.2(2010)$

\title{
The image of a finely holomorphic map is pluripolar
}

\author{
by Armen Edigarian (Kraków), Said El Marzguioui (Amsterdam) \\ and JAN WiEgERINCK (Amsterdam)
}

\begin{abstract}
We prove that the image of a finely holomorphic map on a fine domain in $\mathbb{C}$ is a pluripolar subset of $\mathbb{C}^{n}$. We also discuss the relationship between pluripolar hulls and finely holomorphic functions.
\end{abstract}

1. Introduction. A subset $E \subset \mathbb{C}^{n}$ is said to be pluripolar if for each point $a \in E$ there is an open neighborhood $\Omega$ of $a$ and a function $\varphi(\not \equiv-\infty)$ plurisubharmonic in $\Omega(\varphi \in \operatorname{PSH}(\Omega))$ such that

$$
E \cap \Omega \subset\{z \in \Omega: \varphi(z)=-\infty\} .
$$

It is a fundamental result of Josefson [16] that this local definition is equivalent to the global one, i.e., in this definition one can assume $\varphi$ to be plurisubharmonic in all of $\mathbb{C}^{n}$ with

$$
E \subset\left\{z \in \mathbb{C}^{n}: \varphi(z)=-\infty\right\}
$$

$E$ is called complete pluripolar (in $\mathbb{C}^{n}$ ) if for some plurisubharmonic function $\varphi \in \operatorname{PSH}\left(\mathbb{C}^{n}\right)$, we have $E=\left\{z \in \mathbb{C}^{n}: \varphi(z)=-\infty\right\}$. Unlike the situation in classical potential theory, pluripolar sets often "propagate": it may happen that any PSH function $\varphi$ which is $-\infty$ on a pluripolar set $E$ is automatically $-\infty$ on a larger set. For example, if the $-\infty$ locus of a PSH function $\varphi$ contains a non-polar piece of a complex analytic variety $A$, then the set $\left\{z \in \mathbb{C}^{n}: \varphi(z)=-\infty\right\}$ must contain the whole $A$. However, the structure of pluripolar sets may be much more complicated (cf. [18, 1]). Completeness of pluripolar sets has received growing attention, and in particular cases many results were obtained (see [1, 3] 6, 18, 22, 24, 26]). But our knowledge and understanding of the general situation is fragmentary, and a good characterization of complete pluripolar sets is still lacking, even in the case of graphs of analytic functions.

2010 Mathematics Subject Classification: 32U15, 31C40.

Key words and phrases: finely holomorphic functions, pluripolar sets. 
Around 1980 Fuglede and others developed the theory of so-called finely holomorphic functions, [13. Recently, in [6] Edlund and Jöricke have connected the propagation of the graph of a holomorphic function as a pluripolar set to fine holomorphic continuation of the function.

Theorem 1.1 (Edlund and Jöricke, [6, Theorem 1]). Let $f$ be holomorphic in the unit disc $\mathbb{D} \subset \mathbb{C}$ and let $p \in \partial \mathbb{D}$. Suppose that $f$ has a finely holomorphic continuation $F$ at $p$ to a closed fine neighborhood $V$ of $p$. Then there exists another closed fine neighborhood $V_{1} \subset V$ of $p$ such that the graph $\Gamma_{F}\left(V_{1}\right)$ is contained in the pluripolar hull of $\Gamma_{f}(\mathbb{D})$.

The definition of the pluripolar hull and necessary preliminaries about finely holomorphic functions are presented in Section 2 .

In view of this result, it is reasonable to investigate the connection between finely holomorphic functions and pluripolar sets. Using Fuglede's fundamental work, both in fine potential theory and fine holomorphy, we can give shorter proofs of some known results about pluripolar hulls and obtain our main result, which improves on Theorem 1.1 .

Theorem 1.2. Let $f: U \rightarrow \mathbb{C}^{n}, f(z)=\left(f_{1}(z), \ldots, f_{n}(z)\right)$, be a finely holomorphic map on a finely open set $U \subseteq \mathbb{C}$. Then $f(U)$ is a pluripolar subset of $\mathbb{C}^{n}$. Moreover, if $E$ is a non-polar subset of $U$, then the pluripolar hull of $f(E)$ contains $f(U)$.

Note that in general $U$ may have no Euclidean interior points. The theorem applies e.g. to Borel-type series like

$$
f(z)=\sum_{j=1}^{\infty} \frac{c_{j}}{2^{j}\left(z-a_{j}\right)},
$$

where the $c_{j}$ are very small and $\left\{a_{j}\right\}$ is dense in $\mathbb{C}$. We will give such an example in Section 4.

The next theorem is a simple, precise, and complete interpretation of recent results of the first and third authors (see [3, 4]).

Theorem 1.3. Let $D$ be a domain in $\mathbb{C}$ and let $A$ be a closed polar subset of $D$. Suppose that $f \in \mathcal{O}(D \backslash A)$ and $z_{0} \in A$. Then the following conditions are equivalent:

(1) $\left(\left\{z_{0}\right\} \times \mathbb{C}\right) \cap\left(\Gamma_{f}\right)_{D \times \mathbb{C}}^{*} \neq \emptyset$.

(2) $f$ has a finely holomorphic extension $\tilde{f}$ at $z_{0}$.

Moreover, if one of these conditions holds, then $\left(\left\{z_{0}\right\} \times \mathbb{C}\right) \cap\left(\Gamma_{f}\right)_{D \times \mathbb{C}}^{*}=$ $\left(z_{0}, \tilde{f}\left(z_{0}\right)\right)$.

The proofs of the above results are given in Section 3, Our arguments rely heavily on fine potential theory. Since this theory is not of a very common use in the study of pluripolar sets, we will recall some basic facts in Section 2 . 
Using the same ideas as in the proof of Theorem 1.2 and properties of finely plurisubharmonic functions, a version of Theorem 1.1 for functions of several complex variables has been proved in [8]. We pay some attention to this development at the end of Section 3 . In the final section we discuss some consequences of Theorem 1.2 and some open problems.

\section{Preliminaries}

2.1. Pluripolar hulls. Let $\Omega \subset \mathbb{C}^{n}$ be an open set and let $E \subset \Omega$ be a pluripolar set in $\mathbb{C}^{n}$. The pluripolar hull of $E$ relative to $\Omega$ is the set

$$
E_{\Omega}^{*}=\left\{z \in \Omega: \text { for all } \varphi \in \operatorname{PSH}(\Omega):\left.\varphi\right|_{E}=-\infty \Rightarrow \varphi(z)=-\infty\right\} .
$$

The notion of pluripolar hull was first introduced and studied by Zeriahi in [25]. The paper [19] of Levenberg and Poletsky contains a more detailed study of this notion.

Let $f$ be a holomorphic function in an open set $\Omega \subseteq \mathbb{C}^{n}$. We denote by $\Gamma_{f}(\Omega)$ the graph of $f$ over $\Omega$,

$$
\Gamma_{f}(\Omega)=\{(z, f(z)): z \in \Omega\} .
$$

It is immediate that $\Gamma_{f}(\Omega)$ is a pluripolar subset of $\mathbb{C}^{n+1}$. The pluripolar hulls of the graphs of holomorphic functions were studied in several papers (see [3, 6, 22, 24, 26]).

Of particular interest for our present considerations is the following (see [3, 4]).

Theorem 2.1 (Edigarian and Wiegerinck). Let $D$ be a domain in $\mathbb{C}$ and let $A$ be a closed polar subset of $D$. Suppose that $f \in \mathcal{O}(D \backslash A)$ and that $z_{0} \in A$. Then the following conditions are equivalent:

(i) $\left(\left\{z_{0}\right\} \times \mathbb{C}\right) \cap\left(\Gamma_{f}\right)_{D \times \mathbb{C}}^{*} \neq \emptyset$.

(ii) the set $\{z \in D \backslash A:|f(z)| \geq R\}$ is thin at $z_{0}$ for some $R>0$.

2.2. Fine potential theory. In this subsection we gather some definitions and known results from fine potential theory that we will need later on.

The fine topology on an open set $\Omega$ is the weakest topology on $\Omega$ making all subharmonic functions continuous. If $\Omega_{1} \subset \Omega_{2}$ are domains, then the fine topology on $\Omega_{1}$ coincides with the restriction of the fine topology in $\Omega_{2}$ to $\Omega_{1}$. The following results, except (iv) which is obvious, are due to Fuglede and can be found in [9, Chapters III, IV].

Proposition 2.2.

(i) The fine topology is locally connected.

(ii) Every usual domain is also a fine domain. 
(iii) If $U$ is a fine domain and $E$ is a polar set, then $U \backslash E$ is a fine domain, in particular it is connected.

(iv) The fine topology has a neighborhood basis consisting of fine neighborhoods that are Euclidean compact.

The fine topology has no infinite compact sets and is not Lindelöf. However, the following property can serve as a replacement (see e.g. [2, p. 181].

TheOREM 2.3 (Quasi-Lindelöf property). An arbitrary union of finely open subsets of $\mathbb{C}$ differs from a suitable countable subunion by at most a polar set.

We now formulate the definitions and results concerning fine potential theory that we will use in the present paper. All of these, the proofs, and much more can be found in [9]. All are quite natural in comparison with the classical situation. First we give the definitions.

Definition 2.4. A function $\varphi: U \rightarrow[-\infty,+\infty$ [ defined on a finely open set $U \subseteq \mathbb{C}$ is said to be finely hypoharmonic if $\varphi$ is finely upper semicontinuous and if

$$
\varphi(z) \leq \int \varphi d \varepsilon_{z}^{\mathbb{C} \backslash V}, \quad \forall z \in V \in \mathcal{B}(U) .
$$

(It is part of the requirement that the integral exists.) $\varphi$ is finely subharmonic if, moreover, $\varphi$ is finite on a finely dense subset of $U$.

Here $\mathcal{B}(U)$ denotes the class of all finely open sets $V$ of compact closure $\bar{V}$ (in the usual topology) contained in $U$, and $\varepsilon_{z}^{\mathbb{C} \backslash V}$ is the swept-out of the Dirac measure $\varepsilon_{z}$ onto $\mathbb{C} \backslash V$. It is carried by the fine boundary $\partial_{\mathrm{f}} V$ of $V$. This swept-out measure boils down to the usual harmonic measure if $V$ is a usual open set.

THEOREM 2.5 .

(i) Finely subharmonic functions on a finely open set $\Omega$ form a convex cone that is stable under pointwise supremum for finite families, and closed under finely locally uniform convergence.

(ii) A pointwise infimum of a downward directed family of finely subharmonic functions in a fine domain $\Omega$ is either finely subharmonic, or identically $-\infty$.

(iii) A finely subharmonic function $f$ on a finely open set $\Omega$ has a finely subharmonic restriction to every finely open subset of $\Omega$. Conversely, suppose that $f$ is finely subharmonic in some fine neighborhood of each point of $\Omega$. Then $f$ is finely subharmonic in $\Omega$, i.e., finely subharmonic functions have the sheaf property. 
Proposition 2.6 ([10]). In a usual open set in $\mathbb{C}$ finely subharmonic functions are just subharmonic ones, and the restriction of a usual subharmonic function to a finely open set is finely subharmonic.

TheOREM 2.7. Let $h: U \rightarrow[-\infty,+\infty[$ be a finely hypoharmonic function on a fine domain $U \subset \mathbb{C}$. Then either the set $\{z \in U: h(z)=-\infty\}$ is a polar subset of $U$ and $h$ is finely subharmonic, or $h \equiv-\infty$.

2.3. Finely holomorphic functions. Shortly after the establishment of fine potential theory several authors turned their attention to developing the analog of holomorphic functions on a fine domain (see [13, 14] and the references therein). Fuglede's paper [13] is our main reference for what follows.

For a compact set $K$ in $\mathbb{C}$, we denote by $R(K)$ the uniform closure on $K$ of the set of rational functions with poles outside $K$. By Runge's theorem one can just as well take the closure of the set of functions holomorphic in a neighborhood of $K$.

Definition 2.8. Let $U$ be a finely open set in $\mathbb{C}$. A function $f: U \rightarrow \mathbb{C}$ is called finely holomorphic if every point of $U$ has a compact (in the usual topology) fine neighborhood $K \subset U$ such that the restriction $\left.f\right|_{K}$ belongs to $R(K)$.

As we shall see below, finely holomorphic functions share many properties with ordinary holomorphic functions. We will now assemble the results we will need.

Theorem 2.9. A function $f: U \rightarrow \mathbb{C}$ defined in a finely open set $U \subseteq \mathbb{C}$ is finely holomorphic if and only if every point of $U$ has a fine neighborhood $V \subseteq U$ in which $f$ coincides with the Cauchy-Pompeiu transform of some compactly supported function $\varphi \in L^{2}(\mathbb{C})$ with $\varphi=0$ a.e. in $V$ :

$$
f(z)=\int_{\mathbb{C}} \frac{1}{z-\zeta} \varphi(\zeta) d \lambda(\zeta), \quad z \in V .
$$

THEOREM 2.10. A finely holomorphic function on a Euclidean open set is holomorphic in the usual sense.

THEOREM 2.11.

(i) A finely holomorphic function $f$ on a fine domain has at most countably many zeros (unless $f \equiv 0$ ).

(ii) A finely holomorphic function $f$ is infinitely finely differentiable, and all its fine derivatives $f^{(n)}$ are finely holomorphic.

(iii) Let $f$ be finely holomorphic in a finely open set $U \subset \mathbb{C}$. Suppose that the fine derivative $f^{\prime}$ of $f$ does not vanish at some point $z_{0} \in U$. Then one can find a finely open neighborhood $W \subseteq U$ of $z_{0}$ such 
that $\left.f\right|_{W}: W \rightarrow f(W)$ is bijective and the inverse function $f^{-1}$ is finely holomorphic in the finely open set $f(W)$.

(iv) The composition of finely holomorphic functions is finely holomorphic where it is defined.

(v) Let $U$ be finely open and $z_{0} \in U$. If $f$ is finely holomorphic on $U \backslash\left\{z_{0}\right\}$ and bounded in a punctured fine neighborhood of $z_{0}$, then $f$ extends as a finely holomorphic function to $U$.

3. Pluripolarity of finely holomorphic curves. A finely holomorphic curve is a pair $(U, f)$ where $U$ is a fine domain and $f=\left(f_{1}, \ldots, f_{n}\right): U \rightarrow \mathbb{C}^{n}$ is a finely holomorphic map. As usual we will identify a curve with its image.

Lemma 3.1. Let $U \subseteq \mathbb{C}$ be a fine domain, and let $f: U \rightarrow \mathbb{C}^{n}, f(z)=$ $\left(f_{1}(z), \ldots, f_{n}(z)\right)$, be a finely holomorphic map. Suppose that $h: \mathbb{C}^{n} \rightarrow$ $[-\infty,+\infty[$ is a plurisubharmonic function. Then the function $h \circ f$ is $e i-$ ther finely subharmonic on $U$ or $\equiv-\infty$.

Proof. First, we assume that $h$ is everywhere finite and continuous. Let $a \in U$. Definition 2.8 gives us a compact (in the usual topology) fine neighborhood $K$ of $a$ in $U$, and $n$ sequences $\left(f_{j}^{k}\right)_{k \geq 0}, j=1, \ldots, n$, of holomorphic functions defined in Euclidean neighborhoods of $K$ such that

$$
\left.\left.f_{j}^{k}\right|_{K} \rightarrow f_{j}\right|_{K}, \quad j=1, \ldots, n, \quad \text { uniformly. }
$$

Clearly, $\left(f_{1}^{k}, \ldots, f_{n}^{k}\right)$ converges uniformly on $K$ to $\left(f_{1}, \ldots, f_{n}\right)$. Since $h$ is continuous, the sequence $h\left(f_{1}^{k}, \ldots, f_{n}^{k}\right)$, of finite continuous subharmonic functions, converges uniformly to $h\left(f_{1}, \ldots, f_{n}\right)$ on $K$. According to Theorem 2.5 (i), $h\left(f_{1}, \ldots, f_{n}\right)$ is finely subharmonic in the fine interior of $K$.

Suppose now that $h$ is arbitrary. We can assume that the fine interior of $K$ is finely connected. Let $\left(h_{m}\right)_{m \geq 0}$ be a decreasing sequence of continuous plurisubharmonic functions which converges (pointwise) to $h$. By the first part of the proof, $h_{m}\left(f_{1}, \ldots, f_{n}\right)$ is a decreasing sequence of finely subharmonic functions in the fine interior of $K$. The limit function $h\left(f_{1}, \ldots, f_{n}\right)$ is by Theorem 2.5(ii) finely subharmonic or identically $-\infty$ in the fine interior of $K$. The sheaf property (Theorem 2.5(iii)) implies that $h\left(f_{1}, \ldots, f_{n}\right)$ is indeed finely subharmonic in all of $U$ or is identically equal to $-\infty$.

REMARK 3.2. The above lemma was also independently proved by Fuglede.

Lemma 3.3. Let $f: U \rightarrow \mathbb{C}^{n}, f(z)=\left(f_{1}(z), \ldots, f_{n}(z)\right)$, be a finely holomorphic map on a fine domain $U \subset \mathbb{C}$ which contains a disc with positive radius. Then $f(U)$ is a pluripolar subset of $\mathbb{C}^{n}$.

We denote by $B(a, r)$ the open disc in $\mathbb{C}$ with center $a$ and radius $r>0$. 
Proof. Let $B(a, \delta) \subset U$ be a small disc in $U$. Since $f$ is a holomorphic map on $B(a, \delta)$ (Theorem 2.10), $f(B(a, \delta))$ is a pluripolar subset of $\mathbb{C}^{n}$. By Josefson's theorem there exists a plurisubharmonic function $h \in \operatorname{PSH}\left(\mathbb{C}^{n}\right)$ $(\not \equiv-\infty)$ such that $h\left(f_{1}(z), \ldots, f_{n}(z)\right)=-\infty$ for all $z \in B(a, \delta)$. According to Lemma 3.1, the function $g(z)=h\left(f_{1}(z), \ldots, f_{n}(z)\right)$ is finely subharmonic on $U$ or $\equiv-\infty$. Since it is $-\infty$ on a non-polar subset of $U$, it must be identically $-\infty$ on $U$ by Theorem 2.7. Hence $\left.h\right|_{f(U)}=-\infty$, and so $f(U)$ is pluripolar.

Proposition 3.4. Let $f: U \rightarrow \mathbb{C}^{n}, f(z)=\left(f_{1}(z), \ldots, f_{n}(z)\right)$, be a finely holomorphic map on a finely open subset $U \subseteq \mathbb{C}$. Then the graph $\Gamma_{f}(U)$ is a pluripolar subset of $\mathbb{C}^{n+1}$.

Proof. Since the fine topology is locally connected (Proposition 2.2, it follows from the quasi-Lindelöf property (Theorem 2.3) that $U$ has at most countably many finely connected components. Because a countable union of pluripolar sets is pluripolar, there is no loss of generality to assume that $U$ is a fine domain. Let $a \in U$. According to Theorem 2.9 there exist a finely open fine neighborhood $V \subset U$ of $a$, and $\varphi_{j} \in L^{2}(\mathbb{C}), j=1, \ldots, n$, with compact support such that $\varphi_{j}=0, j=1, \ldots, n$, a.e. in $V$ and

$$
f_{j}(z)=\int_{\mathbb{C}} \frac{1}{z-\zeta} \varphi_{j}(\zeta) d \lambda(\zeta), \quad z \in V, j=1, \ldots, n .
$$

Because of local connectedness, we can assume that $V$ is finely connected. Let $z_{0} \in V$ and $0<\delta<1$ be such that $a \notin \bar{B}\left(z_{0}, \delta\right)$. Choose a smooth function $\rho$ such that $\rho \equiv 1$ on $B\left(z_{0}, \delta / 2\right)$ and $\rho \equiv 0$ on $\mathbb{C} \backslash B\left(z_{0}, \delta\right)$. We set

$$
f_{j}^{1}(z)=\int_{\mathbb{C}} \frac{\rho(\zeta)}{z-\zeta} \varphi_{j}(\zeta) d \lambda(\zeta), \quad f_{j}^{2}(z)=\int_{\mathbb{C}} \frac{1-\rho(\zeta)}{z-\zeta} \varphi_{j}(\zeta) d \lambda(\zeta),
$$

for $j=1, \ldots, n$. It is clear that $f_{j}^{2}, j=1, \ldots, n$, is holomorphic on $B\left(z_{0}, \delta / 2\right)$ and finely holomorphic on the finely open set $V \cup B\left(z_{0}, \delta / 2\right)$. Since usual domains are also finely connected, $V \cup B\left(z_{0}, \delta / 2\right)$ is finely connected. Now, by Lemma 3.3 , the image of $V \cup B\left(z_{0}, \delta / 2\right)$ under $z \mapsto\left(z, f_{1}^{2}(z), \ldots, f_{n}^{2}(z)\right)$ is a pluripolar subset of $\mathbb{C}^{n+1}$. By Josefson's theorem, there exists a plurisubharmonic function $h \in \operatorname{PSH}\left(\mathbb{C}^{n+1}\right)(\not \equiv-\infty)$ such that

$$
h\left(z, f_{1}^{2}(z), \ldots, f_{n}^{2}(z)\right)=-\infty, \quad \forall z \in V \cup B\left(z_{0}, \delta / 2\right) .
$$

Since $f_{j}^{1}, j=1, \ldots, n$, is holomorphic on $\mathbb{C} \backslash \bar{B}\left(z_{0}, \delta\right)$, the function $g: \mathbb{C}^{n+1}$ $\rightarrow \mathbb{C}^{n+1}$ defined by

$$
g\left(z, w_{1}, \ldots, w_{n}\right)=\left(z, w_{1}-f_{1}^{1}(z), \ldots, w_{n}-f_{n}^{1}(z)\right)
$$

is holomorphic on $\left(\mathbb{C} \backslash \bar{B}\left(z_{0}, \delta\right)\right) \times \mathbb{C}^{n}$. Hence $h \circ g$ is plurisubharmonic on 
$\left(\mathbb{C} \backslash \bar{B}\left(z_{0}, \delta\right)\right) \times \mathbb{C}^{n}$ and clearly not identically $-\infty$. Moreover,

$$
\begin{aligned}
h \circ g\left(z, f_{1}(z), \ldots, f_{n}(z)\right) & =h\left(z, f_{1}^{2}(z), \ldots, f_{n}^{2}(z)\right) \\
& =-\infty, \forall z \in V \cap\left(\mathbb{C} \backslash \bar{B}\left(z_{0}, \delta\right)\right) .
\end{aligned}
$$

This proves that the graph $\left\{\left(z, f_{1}(z), \ldots, f_{n}(z)\right): z \in V \cap\left(\mathbb{C} \backslash \bar{B}\left(z_{0}, \delta\right)\right)\right\}$ is a pluripolar subset of $\mathbb{C}^{n+1}$. Notice that $V \cap\left(\mathbb{C} \backslash \bar{B}\left(z_{0}, \delta\right)\right)$ is a finely open set containing the point $a$. Again, by Josefson's theorem, there exists a plurisubharmonic function $\psi \in \operatorname{PSH}\left(\mathbb{C}^{n+1}\right)$ such that

$$
\psi\left(z, f_{1}(z), \ldots, f_{n}(z)\right)=-\infty, \quad \forall z \in V \cap\left(\mathbb{C} \backslash \bar{B}\left(z_{0}, \delta\right)\right) .
$$

In view of Lemma 3.1 the function $z \mapsto \psi\left(z, f_{1}(z), \ldots, f_{n}(z)\right)$ is finely subharmonic in $U$ or $\equiv-\infty$. Since it is $-\infty$ on the non-polar set $V \cap\left(\mathbb{C} \backslash \bar{B}\left(z_{0}, \delta\right)\right)$, it must be identically $-\infty$ on $U$ by Theorem 2.7. This completes the proof.

For convenience of the reader we repeat the statement of our main result, which we will prove subsequently.

Theorem 3.5. Let $f: U \rightarrow \mathbb{C}^{n}, f(z)=\left(f_{1}(z), \ldots, f_{n}(z)\right)$, be a finely holomorphic map on a finely open subset $U \subseteq \mathbb{C}$. Then $f(U)$ is a pluripolar subset of $\mathbb{C}^{n}$. Moreover, if $E$ is a non-polar subset of $U$, then the pluripolar hull of $f(E)$ contains $f(U)$.

Proof. We may assume that $f_{1}$ is not constant and $U$ is a fine domain. By Theorem 2.11 one can choose a non-empty finely open subset $W \subseteq U$ of $U$ such that $\left.f_{1}\right|_{W}: W \rightarrow f_{1}(W)$ is bijective and $f_{1}^{-1}$ is finely holomorphic in the finely open set $f_{1}(W)$. Now, observe that

$$
\begin{aligned}
f(W) & =\left\{\left(f_{1}(z), \ldots, f_{n}(z)\right): z \in W\right\} \\
& =\left\{\left(w, f_{2}\left(f_{1}^{-1}(w)\right), \ldots, f_{n}\left(f_{1}^{-1}(w)\right)\right): w \in f_{1}(W)\right\} .
\end{aligned}
$$

Since the composition of finely holomorphic functions is finely holomorphic (Theorem 2.11), the map $w \mapsto\left(f_{2}\left(f_{1}^{-1}(w)\right), \ldots, f_{n}\left(f_{1}^{-1}(w)\right)\right)$ is finely holomorphic in $f_{1}(W)$. By Proposition 3.4, the graph

$$
\left\{\left(w, f_{2}\left(f_{1}^{-1}(w)\right), \ldots, f_{n}\left(f_{1}^{-1}(w)\right)\right): w \in f_{1}(W)\right\}=f(W)
$$

is a pluripolar subset of $\mathbb{C}^{n}$. Again, Josefson's theorem ensures the existence of a plurisubharmonic function $h \in \operatorname{PSH}\left(\mathbb{C}^{n}\right)$ such that

$$
h\left(f_{1}(z), \ldots, f_{n}(z)\right)=-\infty, \quad \forall z \in W .
$$

By Lemma 3.1, the function $z \mapsto h\left(f_{1}(z), \ldots, f_{n}(z)\right)$ is either finely subharmonic or identically $-\infty$. Since it is $-\infty$ on the non-polar subset $W \subset U$, we must have $h(f(U))=-\infty$ by Theorem 2.7. Repeating this last argument yields the second statement of Theorem 3.5 .

Proof of Theorem 1.3. (1) $\Rightarrow(2)$. According to Theorem 2.1, there exists $R>0$ such that the set $\{z \in D \backslash A:|f(z)| \geq R\}$ is thin at $z_{0}$. Clearly, 
$U=\{z \in D \backslash A:|f(z)|<R\} \cup\left\{z_{0}\right\}$ is a finely open neighborhood of $z_{0}$. Since $f$ is bounded in $U \backslash\left\{z_{0}\right\}$ and finely holomorphic in $U \backslash\left\{z_{0}\right\}$, Theorem 2.11(v) gives that $f$ has a finely holomorphic extension at $z_{0}$.

$(2) \Rightarrow(1)$. Suppose that $f$ has a finely holomorphic extension $\tilde{f}$ at $z_{0}$. Clearly, $(D \backslash A) \cup\left\{z_{0}\right\}$ is a finely open neighborhood of $z_{0}$. Since polar sets do not separate fine domains (Proposition 2.2 the set $(D \backslash A) \cup\left\{z_{0}\right\}$ is finely connected. Let $h \in \operatorname{PSH}(D \times \mathbb{C})$ be a plurisubharmonic function such that $h(z, f(z))=-\infty$ for all $z \in D \backslash A$. According to Lemma 3.1 the function $z \mapsto h(z, \tilde{f}(z))$ is either finely subharmonic on $(D \backslash A) \cup\left\{z_{0}\right\}$ or $\equiv-\infty$. As it is $-\infty$ on $D \backslash A$, it must be identically $-\infty$ in view of Theorem 2.7. Consequently, $\left(z_{0}, \tilde{f}\left(z_{0}\right)\right) \in\left(\Gamma_{f}\right)_{D \times \mathbb{C}}^{*}$. The last assertion follows from Theorem 5.10 in [4].

4. A Borel-type example. We give an example in the spirit of Borel to which the theory applies. It exhibits a finely holomorphic function on a fine domain which is a dense subset of $\mathbb{C}$ with empty Euclidean interior. Our point is to show that the study of quite natural series in connection with pluripolarity is fruitfully done in the framework of fine holomorphy.

EXAMPle 4.1. Let $\left\{a_{j}\right\}_{j=1}^{\infty}$ be a dense sequence in $\mathbb{C}$ with $\left|a_{j}\right|<j$ for all $j$. Let $r_{j}=2^{-j}$. Then $\bigcup_{j=1}^{\infty} B\left(a_{j}, r_{j}\right)$ has finite area, and its circular projection $z \mapsto|z|$ has finite length. Next, define subharmonic functions $g_{j}(z)=\log \left|z-a_{j}\right|-3 j$ and $u_{n}$ by

$$
u_{n}(z)=\sum_{j=n}^{\infty} j^{-3} g_{j}(z) .
$$

The terms in the series (2) are subharmonic, and negative for $|z|<k$ as soon as $j \geq k$. Hence $u_{n}$ represents a subharmonic function. Let

$$
D=\left(\bigcup_{n}\left\{u_{n}>-10\right\}\right) \backslash\left\{a_{1}, a_{2}, \ldots\right\} .
$$

We claim that $D=\left\{u_{1}>-\infty\right\}$. Indeed, let $z_{0} \in \bigcup_{n}\left\{u_{n}>-\infty\right\} \backslash$ $\left\{a_{1}, a_{2}, \ldots\right\}$. Then there exists a natural number $k$ such that $\left|z_{0}\right|<k$ and $u_{k}>-\infty$. Since, as mentioned before, all the terms of the series $u_{k}\left(z_{0}\right)$ are negative, a suitable tail, say $u_{N}\left(z_{0}\right)$, will be very close to 0 . In other words, $z_{0} \in\left\{u_{N}>-10\right\}$. Hence $z_{0} \in D$ and therefore $D=\bigcup_{n}\left\{u_{n}>-\infty\right\} \backslash$ $\left\{a_{1}, a_{2}, \ldots\right\}$. Consequently,

$$
\mathbb{C} \backslash D=\bigcap_{n=1}^{\infty}\left\{u_{n}=-\infty\right\} \cup\left\{a_{1}, a_{2}, \ldots\right\} .
$$

Since $\left\{u_{k_{1}}=-\infty\right\} \backslash\left\{a_{1}, a_{2}, \ldots\right\}=\left\{u_{k_{2}}=-\infty\right\} \backslash\left\{a_{1}, a_{2}, \ldots\right\}$ for any natural 
numbers $k_{1}$ and $k_{2}$, we conclude that

$$
\mathbb{C} \backslash D=\left\{u_{1}=-\infty\right\} \cup\left\{a_{1}, a_{2}, \ldots\right\}=\left\{u_{1}=-\infty\right\} .
$$

This proves the claim. In particular, by Proposition 2.2, $D$ is a fine domain.

For every $j$ there exists $0<c_{j}<1$ such that if $\left|z-a_{j}\right|<c_{j}$, then $u_{n}(z)<-11$ for $n \leq j$. Indeed, $\sum_{k>j} k^{-3} g_{k}(z)<0$, while

$$
\sum_{k=n}^{j-1} k^{-3} g_{k}(z)<\log j \sum_{k=n}^{j-1} k^{-3}<10 \log j,
$$

so it suffices to take $c_{j}=j^{-11 j^{3}}$.

Next we define a function on $D$ by

$$
f(z)=\sum_{j=1}^{\infty} \frac{c_{j}}{2^{j}\left(z-a_{j}\right)} .
$$

We claim that $f$ is finely holomorphic on $D$. Indeed, let $z_{0} \in D$. For every $m$ a suitable tail of the series (3) is uniformly convergent on the compact set $K=\left\{|z| \leq 2\left|z_{0}\right|\right\} \backslash \bigcup_{j \geq m} B\left(a_{j}, c_{j}\right)$. Now if $z_{0} \in D$, then $z_{0}$ belongs to the finely open set $\left\{u_{m}>-10\right\}$ for some $m$. Hence, $\left|z_{0}-a_{j}\right|>c_{j}$ for all $j \geq m$, and $K$ is a fine neighborhood of $z_{0}$.

Application of Theorem 1.2 implies that the graph $\Gamma_{f}(D)$ is a pluripolar set. The theorem also shows that for a set $E \subset D$ of positive capacity, e.g., a circle in $\left\{u_{1}>-10\right\}$,

$$
\Gamma_{f}(D) \subset\left(\Gamma_{f}(E)\right)_{\mathbb{C}^{2}}^{*}
$$

Even for this example there are many questions left open. We have no description of the maximal domain $D_{0}$ to which $f$ extends as a finely holomorphic function, and we do not know if $\Gamma_{f}\left(D_{0}\right)=\left(\Gamma_{f}(E)\right)_{\mathbb{C}^{2}}^{*}$, as one may expect in view of [4].

5. Concluding remarks and open questions. We now discuss some applications and open problems. Let $E \subset \mathbb{C}^{n}$ be a pluripolar set and $E_{\mathbb{C}^{n}}^{*}$ its pluripolar hull. It follows from the arguments used before that if $E$ hits a finely holomorphic curve $f(U)$ in some "non-small" set, then $E_{\mathbb{C}^{n}}^{*}$ contains all the points of $f(U)$. Namely, we have the following.

Proposition 5.1. Let $f: U \rightarrow \mathbb{C}^{n}$ be a finely holomorphic map on a fine domain $U \subset \mathbb{C}$ and let $E \subset \mathbb{C}^{n}$ be a pluripolar set. If $f(U) \cap E \neq \emptyset$ and $f^{-1}(f(U) \cap E)$ is non-polar, then $f(U) \subset E_{\mathbb{C}^{n}}^{*}$.

Proof. Let $h \in \operatorname{PSH}\left(\mathbb{C}^{n}\right)$ be a plurisubharmonic function such that $h(z)$ $=-\infty$ for all $z \in E$. By Lemma 3.1, $h \circ f$ is either finely subharmonic on $U$ or $\equiv-\infty$. As it is $-\infty$ on $f^{-1}(f(U) \cap E)$, it must be identically $-\infty$ on $U$ by Theorem 2.7. Therefore $f(U) \subset E_{\mathbb{C}^{n}}^{*}$. 
The conclusion of the above proposition remains valid if one assumes that $E$ contains merely the "boundary of a finely holomorphic curve".

Proposition 5.2. Let $f$ and $E$ be as above. If $f$ extends by fine continuity to the fine boundary $\partial_{\mathrm{f}} U$ of $U$ and $f\left(\partial_{\mathrm{f}} U\right) \subset E$, then $f(U) \subset E_{\mathbb{C}^{n}}^{*}$.

Proof. Let $h \in \operatorname{PSH}\left(\mathbb{C}^{n}\right)$ be plurisubharmonic function such that $h(z)=$ $-\infty$ for all $z \in E$. Let $a \in \partial_{\mathrm{f}} U$. By assumption, $f$ has a fine limit at $a$. Using Cartan's theorem (cf. [15, Theorem 10.15]), one can easily find a finely open neighborhood $V_{a}$ of $a$ such that the usual $\operatorname{limit} \lim _{z \rightarrow a, z \in V_{a} \cap U} f(z)$ exists and is equal to $f(a)$. Let $M>0$. Since $h$ is upper semicontinuous, the set $\left\{z \in \mathbb{C}^{n}: h(z)<-M\right\}$ is open. As $f(a) \in\left\{z \in \mathbb{C}^{n}: h(z)<-M\right\}$, one can find a positive number $\delta_{a}>0$ such that

$$
f(w) \in\left\{z \in \mathbb{C}^{n}: h(z)<-M\right\}, \quad \forall w \in B\left(a, \delta_{a}\right) \cap V_{a} \cap U .
$$

Consequently,

$$
\mathrm{f}_{z \rightarrow a, z \in U} \limsup h(f(z))=\mathrm{f}_{z \rightarrow a, z \in V_{a} \cap U} h(f(z))<-M, \quad \forall a \in \partial_{\mathrm{f}} U,
$$

where f-limsup denotes the upper limit with respect to the fine topology. As $h \circ f$ is a finely hypoharmonic function on $U$ (see Lemma 3.1 and its proof), the fine boundary maximum principle (cf. [10, Theorem 2.3]) shows that $h \circ f(z)<-M$ for all $z \in U$. Since $M$ was arbitrary, we conclude that $h \circ f(U)=-\infty$. This proves the proposition.

Our results reveal a very close relationship between the pluripolar hulls of the graphs of holomorphic functions and the theory of finely holomorphic functions (see also [6]). This leads naturally to the following fundamental problem.

Problem 1. Let $f: \Omega \rightarrow \mathbb{C}$ be a holomorphic function on a simply connected open subset $\Omega \subset \mathbb{C}$. Suppose that the graph $\Gamma_{f}(\Omega)$ is not complete pluripolar. Must then $\left(\Gamma_{f}(\Omega)\right)_{\mathbb{C}^{2}}^{*} \backslash \Gamma_{f}(\Omega)$ have a fine analytic structure? That is, for $z \in\left(\Gamma_{f}(\Omega)\right)_{\mathbb{C}^{2}}^{*} \backslash \Gamma_{f}(\Omega)$, must there exist a finely holomorphic curve passing through $z$ and contained in $\left(\Gamma_{f}(\Omega)\right)_{\mathbb{C}^{2}}^{*} \backslash \Gamma_{f}(\Omega)$ ?

Obviously, a positive answer to the above problem would, in particular, solve the following problem posed in [6].

Problem 2. Let $f$ be a holomorphic function in the unit disc $\mathbb{D}$. Suppose that $\left(\Gamma_{f}(\mathbb{D})\right)_{\mathbb{C}^{2}}^{*}$ is the graph of some function $\mathcal{F}$. Is $\mathcal{F}$ a finely holomorphic continuation of $f$ ?

It was proved in [1] that one cannot detect "pluripolarity" via intersection with one-dimensional complex analytic varieties. Since there are, roughly speaking, many more finely holomorphic curves in $\mathbb{C}^{n}$ than analytic varieties, one can naturally pose the following 
Problem 3. Let $K$ be a compact set in $\mathbb{C}^{n}$ and suppose that $f^{-1}(K \cap$ $f(U))$ is a polar subset of $U$ (or empty) for any finely holomorphic curve $f: U \rightarrow \mathbb{C}^{n}$. Must $K$ be a pluripolar subset of $\mathbb{C}^{n}$ ?

Finally, in view of Theorem 2.7, it is natural to ask

PROBLEM 4. Is every finely pluripolar set pluripolar?

Acknowledgments. Part of this research was carried out while the second author was visiting the mathematics department at Copenhagen University; he would like to thank the department for its hospitality, and express his gratitude to Professor Bent Fuglede for his invitation and several helpful and interesting discussions.

This work was supported by Research Grant No. 1 PO3A 00528 of the Polish Ministry of Science (2005-2008).

\section{References}

[1] D. Coman, N. Levenberg and E. A. Poletsky, Smooth submanifolds intersecting any analytic curve in a discrete set, Math. Ann. 332 (2005), 55-65.

[2] J. L. Doob, Classical Potential Theory and Its Probabilistic Counterpart, Springer, Berlin, 1984.

[3] A. Edigarian and J. Wiegerinck, The pluripolar hull of the graph of a holomorphic function with polar singularities, Indiana Univ. Math. J. 52 (2003), 1663-1680.

[4] - - - Determination of the pluripolar hull of graphs of certain holomorphic functions, Ann. Inst. Fourier (Grenoble) 54 (2004), 2085-2104.

[5] T. Edlund, Pluripolar sets and pluripolar hulls, Uppsala Dissertations Math. 41 (2005).

[6] T. Edlund and B. Jöricke, The pluripolar hull of a graph and fine analytic continuation, Ark. Mat. 44 (2006), 39-60.

[7] S. El Marzguioui and J. Wiegerinck, The pluri-fine topology is locally connected, Potential Anal. 25 (2006), 283-288.

[8] - - - Connectedness in the Pluri-fine Topology, in: A. Aytuna et al., Functional Analysis and Complex Analysis, Contemp. Math. 481, Amer. Math. Soc., 2009, 105-116.

[9] B. Fuglede, Finely Harmonic Functions, Lecture Notes in Math. 289, Springer, Berlin, 1972.

[10] - Fonctions harmoniques et fonctions finement harmoniques, Ann. Inst. Fourier (Grenoble) 24 (1974), no. 4, 77-91.

[11] -, Asymptotic paths for subharmonic functions, Math. Ann. 213 (1975), 261-274.

[12] - Finely harmonic mappings and finely holomorphic functions, Ann. Acad. Sci. Fenn. 2 (1976), 113-127.

[13] - Sur les fonctions finement holomorphes, Ann. Inst. Fourier (Grenoble) 31 (1981), no. $4,57-88$.

[14] —, Finely holomorphic functions - a survey, Rev. Roumaine Math. Pures Appl. 33 (1988), 283-295.

[15] L. L. Helms, Introduction to Potential Theory, Pure Appl. Math. 22, Wiley-Interscience, New York, 1969. 
[16] B. Josefson, On the equivalence between locally polar and globally polar sets for plurisubharmonic functions on $\mathbb{C}^{n}$, Ark. Mat. 16 (1978), 109-115.

[17] M. Klimek, Pluripotential Theory, London Math. Soc. Monogr. 6, Clarendon Press, Oxford, 1991.

[18] N. Levenberg, On an example of Wermer, Ark. Mat. 26 (1988), 155-163.

[19] N. Levenberg and E. A. Poletsky, Pluripolar hulls, Michigan Math. J. 46 (1999), $151-162$.

[20] N. Levenberg and Z. Słodkowski, Pseudoconcave pluripolar sets in $\mathbb{C}^{2}$, Math. Ann. 312 (1998), 429-443.

[21] N. V. Shcherbina, Pluripolar graphs are holomorphic, Acta Math. 194 (2005), 203216.

[22] J. Siciak, Pluripolar sets and pseudocontinuation, in: Complex Analysis and Dynamical Systems II (Nahariya, 2003), Contemp. Math. 382, Amer. Math. Soc., Providence, RI, 2005, 385-394.

[23] J. Wermer, Polynomially convex hulls and analyticity, Ark. Mat. 20 (1982), 129-135.

[24] J. Wiegerinck, Graphs of holomorphic functions with isolated singularities are complete pluripolar, Michigan Math. J. 47 (2000), 191-197.

[25] A. Zeriahi, Ensembles pluripolaires exceptionnels pour la croissance partielle des fonctions holomorphes, Ann. Polon. Math. 50 (1989), 81-91.

[26] W. Zwonek, A note on pluripolar hulls of graphs of Blaschke products, Potential Anal. 22 (2005), 195-206.

Armen Edigarian

Institute of Mathematics

Jagiellonian University

Łojasiewicza 6

30-348 Kraków, Poland

E-mail: Armen.Edigarian@im.uj.edu.pl
Said El Marzguioui, Jan Wiegerinck

KdV Institute for Mathematics University of Amsterdam PO box 94248

1090 GE Amsterdam, The Netherlands E-mail: smarzgui@science.uva.nl j.j.o.o.wiegerinck@uva.nl

Received 2.3.2009

and in final form 28.8.2009 
